\title{
THE EINSTEIN-DIRAC EQUATION ON SASAKIAN 3-MANIFOLDS
}

\author{
FLORIN ALEXANDRU BELGUN
}

\begin{abstract}
We prove that a Sasakian 3-manifold admitting a non-trivial solution to the Einstein-Dirac equation has necessarily constant scalar curvature. In the case when this scalar curvature is non-zero, their classification follows then from a result by Th. Friedrich and E.C. Kim. We also prove that a scalar-flat Sasakian 3-manifold admits no local Einstein spinors.
\end{abstract}

\section{INTRODUCTION}

On a (pseudo-) Riemannian spin manifold $(M, g)$, a solution of the Einstein-Dirac equation is an eigenspinor $\psi$ of the Dirac operator (i.e.

$$
D \psi=\lambda \psi
$$

for a real constant $\lambda$ ) satisfying to the Einstein equation

$$
\text { Ric }-\frac{1}{2} S g= \pm \frac{1}{4} T_{\psi}
$$

where Ric, resp. $S$, are Ricci and the scalar curvature of the manifold, and $T_{\psi}$ is the energy-momentum tensor given by the spinor $\psi$ by the formula

$$
T_{\psi}(X, Y):=\left(Y \cdot \nabla_{X} \psi+X \cdot \nabla_{Y} \psi, \psi\right) \forall X, Y \in T M,
$$

where $\nabla$ is the Levi-Civita-spin connection, the dot stands for Clifford multiplication, and $(\cdot, \cdot)$ is the real part of the Hermitian scalar product on the spin bundle $\Sigma$.

The system of equations (1-2), henceforth called the Einstein-Dirac equation, has been considered in Physics for a long time in dimension 4 and Lorentzian signature, as describing the interaction of a particle of spin $\frac{1}{2}$ with the gravitation field [2]. As it has recenlty been shown by Th. Friedrich and E.C. Kim [4], the above equations arise as the Euler-Lagrange equations characterizing the extrema of the functional

$$
W^{\varepsilon}(g, \psi):=\int_{M}\left(S_{g}+\varepsilon\left(\lambda(\psi, \psi)-\left(D^{g} \psi, \psi\right)\right)\right) V_{g},
$$

where $\varepsilon= \pm 1$ and $V_{g}$ is the volume form of the manifold. The coupling of the equations (1) and (2) arises naturally, as the differential identity (coming from the second Bianchi identity) satisfied by the Einstein tensor Ric $-\frac{1}{2} S g$ is automatically satisfied by an energy-momentum tensor of type (3), if $\psi$ is an eigenspinor of the Dirac operator [4].

In the above cited paper, Th. Friedrich and E.C. Kim constructed families of solutions of (1-2) - also called Einstein spinors - , and one of their methods relies on the

Date: August 1, 2000.

AMS classification : $53 \mathrm{C} 25,53 \mathrm{C} 27$.

Supported by the SFB 288 of the DFG. 
introduction of the weak Killing equation (for short $W K$ ):

$$
\nabla_{Y} \psi=\frac{n}{2(n-1) S} d S(Y) \psi+\frac{2 \lambda}{(n-2) S} \operatorname{Ric}(Y) \cdot \psi-\frac{\lambda}{n-2} Y \cdot \psi+\frac{1}{2(n-1) S} Y \cdot d S \cdot \psi,
$$

where $n$ is the dimension of the manifold, and $\lambda$ is a real constant, also called the $W K$ number of the spinor $\psi$. They show that any $W K$ spinor with $W K$ number $\lambda$ yields (by multiplication with a constant) an Einstein spinor, of eigenvalue $\lambda$ for the Dirac operator. They obtain, for all $n$, examples of Sasakian manifolds with constant scalar curvature admitting non-trivial $W K$ spinors, therefore also Einstein spinors.

Moreover, in dimension $n=3$, the existence of a nontrivial solution to the EinsteinDirac equation is equivalent, on the open set where $S$ does not vanish, to the existence of a non-trivial $W K$ spinor. It is shown in [4] that, up to a local isometry, there are exactly 3 Sasakian metrics (see below) on 3-manifolds with constant scalar curvature (one of which is the round sphere $S^{3}$ ) admitting non-trivial such spinors.

The aim of this note is to investigate the case of a general Sasakian 3-manifold; we establish the following

Theorem 1. Let $(M, g)$ be a simply-connected 3-dimensional Sasakian manifold. Then it admits nontrivial Einstein spinors $\psi$ with eigenvalue $\lambda$ for the Dirac operator if and only if the scalar curvature $S$ is constant and equal to one of the following values:

(i) $S=6$, and $M$ is locally isometric to the round 3-sphere, $\psi$ is a Killing spinor and $\lambda=\frac{3}{2}$

(ii) $S=1+\sqrt{5}, \lambda=\frac{2+\sqrt{5}}{2}$, and $\psi$ is not a Killing spinor;

(iii) $S=1-\sqrt{5}, \lambda=\frac{2-\sqrt{5}}{2}$, and $\psi$ is not a Killing spinor.

The examples from (i) to (iii) are due to Th. Friedrich and E.C. Kim [4]. The last 2 examples are part of a 1-parameter (modulo homothety) family of metrics on 3-manifolds with constant eigenvalues of the Ricci tensor, which, as shown by Th. Friedrich [3], are the only ones that admit non-trivial Einstein spinors, and do not admit Killing spinors.

The proof of the Theorem is divided in two steps: first (Section 3) we prove (using the $W K$ equation (5)) that, if the scalar curvature is non-vanishing and the manifold admits locally an Einstein spinor, then the scalar curvature is constant; in the second step (Section 4) we prove that, if the scalar curvature of a Sasakian 3-manifold is constantly zero, then there is no Einstein spinor (note that the $W K$ equation is not defined in this case).

Acknowledgements. I am grateful to Th. Friedrich who kindly answered to my questions.

\section{SPINORS ON SASAKIAN 3-MANIFOLDS}

Consider the Clifford algebra bundle $C l(M)$ of a Riemannian oriented 3-manifold $M$, defined as the quotient of the tensor algebra $\otimes M$ by the bilateral ideal generated by the elements

$$
X \otimes Y-Y \otimes X+2 g(X, Y) .
$$

Then $C l(M)$ is isomorphic, as a vector bundle, to the exterior algebra bundle $\Lambda(M)$, therefore its fiber has dimension 8. It is a standard fact [5] that $C l(M)_{x} \otimes \mathbb{C}$ is isomorphic, as a complex algebra, to the direct sum $\operatorname{End}\left(\Sigma_{x}\right) \oplus \operatorname{End}\left(\Sigma_{x}^{\prime}\right)$, for $\Sigma_{x}, \Sigma_{x}^{\prime}$ 2 -dimensional complex vector spaces. These 2 fundamental complex representations of $C L(M)_{x}$, distinguished by the action (equal to the identity on $\Sigma_{x}$ and to minus the 
identity on $\Sigma_{x}^{\prime}$ ) of the central element $V:=e_{1} \cdot e_{2} \cdot e_{3}$ (for $e_{1}, e_{2}, e_{3}$ an oriented orthonormal basis of $T_{x} M$ ) of square 1 , are both isomorphic to the fundamental representation of the group $\operatorname{Spin}(M)_{x} \subset C l(M)_{x}$, which is the universal covering of $S O(M)_{x}$, therefore isomorphic to $S U(2)$.

Convention. We will consider, in this paper, the spinor bundle $\Sigma$, consisting of the fundamental representations of $C l(M)$ on which the volume element $V$ acts like the identity (consideration of the other spinor bundle, on which the element above acts as minus the identity, leads to an equivalent situation; only that the $W K$-number $\lambda$ arising in the $W K$ equation (5) has to be replaced by its opposite).

In all generality, the spinor bundle $\Sigma$, as well as the $\operatorname{Spin}(3) \simeq S U(2)$ principal bundle $\operatorname{Spin}(M)$, double covering the frame bundle $S O(M)$, can only be considered locally. However, as any orientable 3 -manifold is paralelizable, its frame bundle is trivial and the $\operatorname{Spin}(M)$ bundle can be globally constructed: the manifold is spin. Actually, the restriction to spin manifolds is not essential for our purposes, as we consider local solutions of the Einstein-Dirac equation.

As $T M \subset C l(M)$, it acts, by Clifford multiplication, denoted by a dot, on $\Sigma$; this action preserves the complex structure of $\Sigma \simeq \mathbb{C}^{2}$ (which can be seen as the structure induced by the left multiplication by the quaternion $i \in \mathbf{H}$ on $\mathbf{H} \simeq \mathbb{C}^{2}$ ), and $X \cdot \psi$, for $X \in T_{x} M \simeq \operatorname{Im}(\mathbf{H})$ and $\psi \in \Sigma \simeq \mathbf{H}$ can be seen to be isomorphic to the right multiplication with the quaternion $X$ (if we had taken $\Sigma^{\prime}$ for the spinor bundle, it would have been the right multiplication by $-X)$; the orientation of $T_{x} M \simeq \operatorname{Im}(\mathbf{H})$ is supposed to be induced by the oriented basis $i, j, k \in \mathbf{H}$.

Let us consider now the case where $(M, g)$ is a Sasakian manifold.

Definition 1. A Sasakian structure on an oriented 3-manifold $M$ is given by an unitary Killing vector field $T$, such that $J:=\nabla T \in \operatorname{End}\left(T^{\perp}\right)$ is a complex structure on this rank 2 subbundle (i.e., its square is equal to minus the identity). The orientation compatibility is, by definition, such that $T, X, J X$ is an oriented orthonormal basis of $T M$ for any unitary vector $X \in T^{\perp}$.

Remark. The fact that $J X \perp X$ follows from the fact that $T$ is Killing, and the fact that $\nabla T$ can be restricted to $T^{\perp}$ is due to the additionary fact that $T$ is unitary.

We notice that the integral curves of $T$ are geodesics, and the flow of $T$ preserves the complex structure on $Q:=T^{\perp}$, thus a Sasakian metric on a 3-manifold is locally isometric to a real line bundle over a Riemann surface, with the metric given by a metric on the basis $B$ (equivalently by its Kähler form $\omega_{B}$ ), one on the fiber, and a connection on this line bundle such that its curvature is $2 i \omega_{B}[1]$. This latter condition immediately follows from

$$
\nabla_{X} T=J X
$$


as do the following ones:

$$
\begin{aligned}
{[J X, T] } & =J[X, T] \in Q ; \\
R_{T, X} & =-T \wedge X ; \\
R_{T, J X} & =-T \wedge J X ; \\
R_{X, J X} & =-\frac{S-4}{2} X \wedge J X ; \\
d S(T) & =0 ; \\
R i c & =\left(\begin{array}{ccc}
2 & 0 & 0 \\
0 & \frac{S}{2}-1 & 0 \\
0 & 0 & \frac{S}{2}-1
\end{array}\right),
\end{aligned}
$$

where the Ricci tensor has been realized as a $3 \times 3$ matrix using the positive orthonormal basis $\{T, X, J X\}$, and $S$ denotes the scalar curvature.

\section{Proof of the Theorem - the case $S \neq 0$}

Let $\left(M^{3}, g, T\right)$ be a Sasakian 3-manifold, and endow it with the orientation given by the basis $T, X, J X$, for $0 \neq X \in T^{\perp}$, and $J X:=\nabla_{X} T$. Then $M$ is spin, and suppose the Einstein-Dirac equation (1-2) admits a non-trivial solution $\psi \in C^{\infty}(\Sigma)$. Consider first the open set of $M$ where the scalar curvature $S$ does not vanish. It foolows then from [4] that, on this open set, $\psi$ must be a solution of the $W K$ equation (5):

$$
\nabla_{Y} \psi=\frac{3}{4 S} d S(Y) \psi+\frac{2 \lambda}{S} \operatorname{Ric}(Y) \cdot \psi-\lambda Y \cdot \psi+\frac{1}{4 S} Y \cdot d S \cdot \psi
$$

We can choose a unitary vector field $X \perp T$ on a neighborhood of a point $x_{0} \in M$, such that:

$$
\begin{aligned}
{[X, T] } & =0 \text { everywhere; } \\
\left(\nabla_{X} J X\right)_{x_{0}} & =-T .
\end{aligned}
$$

We re-write the WK equation for the special cases $Y=T$ and $Y=X$, replacing $d S$ by $d S(X) X+d S(J X) J X$ (recall that $d S(T)=0$ ):

$$
\begin{aligned}
& \nabla_{T} \psi=\frac{(4-S) \lambda}{S} T \cdot \psi+\frac{d S(J X)}{4 S} X \cdot \psi-\frac{d S(X)}{4 S} J X \cdot \psi \\
& \nabla_{X} \psi=\frac{d S(X)}{2 S} \psi-\frac{d S(J X)}{4 S} T \cdot \psi-\frac{2 \lambda}{S} X \cdot \psi .
\end{aligned}
$$

We want to prove that the existence of a non-trivial local solution of this equation implies that the scalar curvature $S$ is constant.

Suppose that $\psi$ is such a solution; then it is non-vanishing [4] and thus $\{\psi, T \cdot \psi, X$. $\psi, J X \cdot \psi\}$ is an orthogonal basis for $\Sigma$. We are going to compute $R_{T, X} \cdot \psi$ using the WK-equations for $\psi(15)$, and compare this with the known value of it (7-11), i.e. $-(T \wedge X) \cdot \psi=-1 / 2 J X \cdot \psi$. We will develop it on the above mentioned basis of $\Sigma$, and will actually be concerned only with the coefficient of $T \cdot \psi$. We define $\eta: \Sigma \rightarrow \mathbb{R}$ such that $\eta(\psi)=\eta(X \cdot \psi)=\eta(J X \cdot \psi)=0$ and $\eta(T \cdot \psi)=1$; then the coefficient we are looking to identify at $\nabla_{T} \nabla_{X} \psi$ and $\nabla_{X} \nabla_{T} \psi$ is $\eta\left(\nabla_{T} \nabla_{X} \psi\right)$, respectively $\eta\left(\nabla_{X} \nabla_{T} \psi\right)$ (they need to be equal because $R_{T, X}$ - see (7-11) - acts on $\psi$ as (a constant times) the Clifford multiplication by $J X)$.

Let us write the equations (15) as

$$
\begin{aligned}
& \nabla_{T} \psi=A \psi:=a \psi+a^{T} T \cdot \psi+a^{X} X \cdot \psi+a^{J X} J X \cdot \psi ; \\
& \nabla_{X} \psi=B \psi:=b \psi+b^{T} T \cdot \psi+b^{X} X \cdot \psi+b^{J X} J X \cdot \psi .
\end{aligned}
$$


As we have that $b^{T}$ is constant along $T$,

$$
\eta\left(\nabla_{T} \nabla_{X} \psi\right)=\eta(B A \psi)=b a^{T}+b^{T} a-b^{X} a^{J X}+b^{J X} a^{X},
$$

and, as $a=0$ and $b^{J X}=0$, we get

$$
\eta\left(\nabla_{T} \nabla_{X} \psi\right)=b a^{T}-b^{X} a^{J X} .
$$

On the other hand, we have

$$
\eta\left(\nabla_{X} \nabla_{T} \psi\right)=d a^{T}(X)-a^{J X}+\eta(A B \psi),
$$

where the first term on the r.h.s. comes from the derivation of the coefficient of $T \cdot \psi$ in $\nabla_{T} \psi$, and the second appears because, at the given point $x_{0}$ where we make the computations, $\nabla_{X} J X=-T$ (and $\left.\nabla_{X} X=0\right)$. We get then

$$
\eta\left(\nabla_{X} \nabla_{T} \psi\right)=b a^{T}+b^{X} a^{J X}+d a^{T}(X)-a^{J X} .
$$

By setting $\eta\left(R_{T, X} \cdot \psi\right)=0$, from (19) and (20) we get:

$$
2 b^{X} a^{J X}+d a^{T}(X)-a^{J X}=0,
$$

or, equivalently:

$$
(4 \lambda-16 \lambda+S) \frac{d S(X)}{4 S^{2}}=0 .
$$

We could have started with another vector $X \in T^{\perp}$, and in any other point $x_{0}$, so the above equation should hold everywhere, and for any vector $X$ orthogonal to $T$. If we suppose that $S$ is not constant, then on an open set we have $d S \neq 0$, thus we can find a vector field $X$ such that, on an open set, we have $d S(X) \neq 0$. From (21) we get then that $12 \lambda-S=0$ on an open set, thus $S$ is constant there, contradiction.

It follows then that $S$ is constant everywhere; it is non-zero, thus either it is constant and non-zero, or it is identically zero. In the first case, it has been established by Th. Friedrich and E.C. Kim that only the possibilities listed above in the Theorem exist. In the second case, from the Einstein-Dirac equation it follows [4] that

$$
S=\mp \lambda|\psi|^{2},
$$

hence $\lambda=0$ (as $\psi$ is supposed to be non-trivial).

Thus any 3-dimensional Sasakian manifold with non-zero scalar curvature, admitting a non-trivial solution to the WK equation, has constant scalar curvature.

\section{Proof of the Theorem - CASE $S=0$}

Let $(M, g, T)$ be, as in the prevoius section, a Sasakian 3-manifold, and suppose in addition that its scalar curvature, $S$, is identically zero. All such manifolds are locally isometric, and any compact example is, up to a finite covering, $S^{3}$ equipped with a Berger metric, such that the metric on the fibers of the Hopf fibration $S^{3} \rightarrow \mathbb{C P}^{1}$ bear the usual metric (i.e. are circles of length $2 \pi$ ), and are orthogonal to the horizontal spaces (consisting in the canonical contact structure on $S^{3}$ ), on which the metric comes from a constant $K=\frac{1}{2}$ curvature metric on $\mathbb{C P}^{1}$ (the round metric on $S^{3}$ is induced by a constant $K=2$ curvature metric on $\mathbb{C P}^{1}$ ).

Let $\psi$ be a solution of the Einstein-Dirac equation (1-2); if we suppose it is nontrivial, then it does not vanish on an open set, to which we restrict ourselves from now 
on. We can thus write $\psi=e^{f} \phi$, where $f$ is a real-valued function and $\phi$ an unitary spinor. The Einstein-Dirac equations are then equivalent to

$$
\begin{aligned}
D \phi+d f \cdot \phi & =0, \\
\left(X \cdot \nabla_{Y} \phi+Y \cdot \nabla_{X} \phi, \phi\right) & = \pm 4 e^{-2 f} \operatorname{Ric}(X, Y), \forall X, Y \in T M .
\end{aligned}
$$

If we denote by $A, B, C$ a positive orthonormal basis of $T M$, by developping the first equation above, and by Clifford-multiplying it by $C$, we get

$$
\left(A \cdot \nabla_{B} \phi-B \cdot \nabla_{A} \phi, \phi\right)=d f(C),
$$

from which, using the fact that $\phi$ is unitary, and (23), we obtain the following system of equations (the remaining two are obtained by circular permutations of the one below), which are linear for $\phi$, and one can easily check that they are equivalent to the EinsteinDirac equations:

$$
\nabla_{A} \phi=\mp 2 e^{-2 f} \operatorname{Ric}(A, A) A \cdot \phi-\frac{1}{2} d f(C) B \cdot \phi+\frac{1}{2} d f(B) C \cdot \phi .
$$

We specialize for the basis $T, X, J X$ and we get:

$$
\begin{aligned}
& \nabla_{T} \phi=\mp 4 e^{-2 f} T \cdot \phi \quad+\frac{1}{2} d f(J X) X \cdot \phi \quad-\frac{1}{2} d f(X) J X \cdot \phi \\
& \nabla_{X} \phi=-\frac{1}{2} d f(J X) T \cdot \phi \quad \pm 2 e^{-2 f} X \cdot \phi \quad+\frac{1}{2} d f(T) J X \cdot \phi \\
& \nabla_{J X} \phi=+\frac{1}{2} d f(X) T \cdot \phi \quad-\frac{1}{2} d f(T) X \cdot \phi \quad \pm 2 e^{-2 f} J X \cdot \phi .
\end{aligned}
$$

So $\phi$ satisfies to a linear first order PDE, and can be geometrically interpreted as a parallel section of the spinor bundle for a certain linear connection, defined by the equations above. The only local obstruction to the existence of such a section is thus contained in the curvature of this connection, or, equivalently, such a section $\phi$ exists if and only if the expressions of the type $\nabla_{A} \nabla_{B} \phi-\nabla_{B} \nabla_{A} \phi-\nabla_{[A, B]} \phi$, computed using the equations (25), coincide with the known values of the curvature $R_{A, B} \phi$. We get, after similar computations as in the previous section, the following expressions for the curvature terms:

$$
\begin{aligned}
R_{T, X} \phi= & {\left[-\frac{1}{2} d^{2} f(T, J X) \mp 6 e^{-2 f} d f(X)+\frac{1}{2} d f(T) d f(J X)\right] T \cdot \phi+} \\
& {\left[-\frac{1}{2} d^{2} f(X, J X)+\frac{1}{2} d f(X) d f(J X)\right] X \cdot \phi+} \\
& {\left[\frac{1}{2} d^{2} f(T, T)+\frac{1}{2} d^{2} f(X, X) \pm 6 e^{-2 f}+\frac{1}{2} d f(J X)^{2}-16 e^{-4 f}\right] J X \cdot \phi ; } \\
R_{X, J X} \phi= & {\left[\frac{1}{2} d^{2} f(X, X)+\frac{1}{2} d^{2} f(J X, J X) \mp 12 e^{-2 f}+8 e^{-4 f}+\frac{1}{2} d f(T)^{2}\right] T \cdot \phi+} \\
& {\left[-\frac{1}{2} d^{2} f(T, X)+\frac{1}{2} d f(T) d f(X) \pm 6 e^{-2 f} d f(J X)\right] X \cdot \phi+} \\
& {\left[-\frac{1}{2} d^{2} f(T, J X) \mp 6 e^{-2 f} d f(X)+\frac{1}{2} d f(T) d f(J X)\right] J X \cdot \phi ; } \\
R_{J X, T} \phi= & {\left[-\frac{1}{2} d^{2} f(T, X)+\frac{1}{2} d f(T) d f(X) \pm 6 e^{-2 f} d f(J X)\right] T \cdot \phi+} \\
& {\left[\frac{1}{2} d^{2} f(T, T)+\frac{1}{2} d^{2} f(J X, J X) \pm 6 e^{-2 f}-16 e^{-4 f}+\frac{1}{2} d f(X)^{2}\right] X \cdot \phi+} \\
& {\left[-\frac{1}{2} d^{2} f(X, J X)+\frac{1}{2} d f(X) d f(J X)\right] X \cdot \phi . }
\end{aligned}
$$

We have denoted by $d^{2} f$ the Hessian of $f, d^{2} f(X, Y):=X .(Y . f)-\left(\nabla_{X} Y\right) . f$. This leads to a system of second order equations for the function $f$; if we replace $d f$, each time it appears, with the (closed) 1-form $\theta$, we get, from (26), the following equations for $\theta$ :

$$
\begin{aligned}
\nabla_{T} \theta(X)=\nabla_{X} \theta(T) & = \pm 12 e^{-2 f} \theta(J X)+\theta(T) \theta(X) ; \\
\nabla_{T} \theta(J X)=\nabla_{J X} \theta(T) & =\mp 12 e^{-2 f} \theta(X)+\theta(T) \theta(J X) ; \\
\nabla_{X} \theta(J X)=\nabla_{J X} \theta(X) & =\theta(X) \theta(J X) ;
\end{aligned}
$$




$$
\begin{aligned}
\nabla_{T} \theta(T) & =\mp 24 e^{-2 f}+40 e^{-4 f}+\frac{1}{2}\left[\theta(T)^{2}-\theta(X)^{2}-\theta(J X)^{2}\right]-2 ; \\
\nabla_{X} \theta(X) & = \pm 12 e^{-2 f}-8 e^{-4 f}+\frac{1}{2}\left[-\theta(T)^{2}+\theta(X)^{2}-\theta(J X)^{2}\right]+1 ; \\
\nabla_{J X} \theta(J X) & = \pm 12 e^{-2 f}-8 e^{-4 f}+\frac{1}{2}\left[-\theta(T)^{2}-\theta(X)^{2}+\theta(J X)^{2}\right]+1 .
\end{aligned}
$$

We have two cases: either $d f=\theta$ is identically zero, and then the last 3 equations above lead to a contradiction, or we can restrict ourselves to an open set where it does not vanish, and then we compare again the curvature terms $R_{A, B}$ coming from the equations (27-30) with the known values (7-11).

We compute $\nabla_{T} \nabla_{X} \theta(T)=T \cdot\left(\nabla_{X} \theta(T)\right)$ (as $\left.\nabla_{T} T=0\right)$, then $\nabla_{X} \nabla_{T} \theta(T)=$ $X .\left(\nabla_{T} \theta(T)\right)-\nabla_{J X} \theta(T)$ (recall that $\left.\nabla_{X} T=J X\right)$ from the equations above (and replace $d f$ by $\theta)$; we get:

$$
R_{T, X} \theta(T)=\left(\mp 84 e^{-2 f}+48 e^{-4 f}-1\right) \theta(X) \mp e^{-2 f} \theta(T) \theta(J X) ;
$$

as it has to be identically equal to a constant times $\theta(X)$ (see (7-11)), it follows that $f$ is a constant, or, equivalently, $\theta=0$.

This ends the proof of the Theorem.

\title{
REFERENCES
}

[1] F.A. Belgun, Normal CR structures on compact 3-manifolds, to appear in Math. Z.

[2] R. Bill, J.A. Wheeler, Interaction of neutrinos and gravitational fields, Rev. Mod. Phys. 29 (1957), 465-479.

[3] Th. Friedrich, Solutions of the Einstein-Dirac Equation on Riemannian Manifolds with Constant Scalar Curvature, math.DG/0002182, to appear in J. Geom. Phys.

[4] Th. Friedrich, E.C. Kim, The Einstein-Dirac Equation on Riemanian Spin Manifolds, J. Geom. Phys 33 (2000), 128-172.

[5] H.B. Lawson, M.-L. Michelson, Spin Geometry, Princeton University Press 1989.

\author{
MATHEMATISCHES INSTITUT \\ HUMBOLDT UNIVERSITÄT ZU BERLIN \\ UNTER DEN LINDEN 6, 10099 BERLIN \\ GERMANY \\ e-mail: belgun@mathematik.hu-berlin.de
}

